\section{A case of attempted bilateral self-enucleation in a patient with bipolar disorder}

\author{
Hannah Muniz Castro, ${ }^{1}$ John Alvarez, 2 \\ Robert G. Bota, 2 Marc Yonkers, ${ }^{3}$ \\ Jeremiah $\mathrm{Tao}^{3}$ \\ 1 School of Medicine, University of \\ California, Irvine, CA; ${ }^{2}$ Department of \\ Psychiatry, University of California, \\ Irvine, CA; 3Department of \\ Ophthalmology, Gavin Herbert Eye \\ Institute, CA, USA
}

\begin{abstract}
Attempted and completed self-enucleation, or removal of one's own eyes, is a rare but devastating form of self-mutilation behavior. It is often associated with psychiatric disorders, particularly schizophrenia, substance induced psychosis, and bipolar disorder. We report a case of a patient with a history of bipolar disorder who gouged his eyes bilaterally as an attempt to self-enucleate himself. On presentation, the patient was manic with both psychotic features of hyperreligous delusions and command auditory hallucinations of God telling him to take his eyes out. On presentation, the patient had no light perception vision in both eyes and his exam displayed severe proptosis, extensive conjunctival lacerations, and visibly avulsed extraocular muscles on the right side. An emergency computed tomography scan of the orbits revealed small and irregular globes, air within the orbits, and intraocular hemorrhage. He was taken to the operating room for surgical repair of his injuries. Attempted and completed self-enucleation is most commonly associated with schizophrenia and substance induced psychosis, but can also present in patients with bipolar disorder. Other less commonly associated disorders include obsessive-compulsive disorder, depression, mental retardation, neurosyphilis, Lesch-Nyhan syndrome, and structural brain lesions.
\end{abstract}

\section{Introduction}

Self-enucleation, autoenucleation, or oedipism, is one of the major forms of selfmutilation. While it was previously thought to occur equally in men and women, a recent review revealed that the incidence may be higher in males than females. ${ }^{1}$ Prevalence is reported between 2.8-4.2 in 100,000 and incidence of 1 in 30 million people. ${ }^{2}$ A review of 56 cases found that one third of the cases are bilateral. ${ }^{3}$ The most common underlying motivations for selfenucleation are biblical interpretation, hypersexual or hyperreligious delusions, and paranoia regarding the eye.

The term oedipism was coined after Oedipus Rex, the mythological protagonist in Sophocles' tragedy Oedipus the King, who blinded himself after unknowingly killing his father and marrying his mother. ${ }^{4}$ A commonly cited biblical reference from the New Testament in the Book of Matthew 5:29 is often cited by patients after self inflicting injuries to the eye: If thy right eye causeth thee to stumble, pluck it out, and cast it from thee. ${ }^{5}$ Hyperreligiosity tends to be more common among patients who attempt or complete self-enucleation, as in the patient we report. Approximately half of all cases of self-inflicted eye injuries are associated with religious delusions. ${ }^{6}$

In a recent review of 60 cases of complete and 9 cases of attempted self-enucleation, $43 \%$ of the cases were associated with schizophrenia, $25 \%$ were drug induced, and only $4 \%$ were associated with bipolar disorder. ${ }^{1}$ It is therefore imperative for physicians to be aware of the selfdestructive behavior of patients, especially those with underlying mental disorders or drug abuse.

\section{Case Report}

A 52-year-old Caucasian male with history of bipolar disorder was brought in by his son to the emergency room (ER) following severe bilateral injuries to his eyes as an attempt to self-enucleate. The patient called his son to his bedroom after attempting to manually pull his eyes out with his fingers because he was instructed to do so by God and felt the need to cleanse myself from sin. The patient was transferred to the ER by ambulance and appeared calm. The son reports that the patient had been acting strange and displayed decreased appetite and increased wakefulness. He was noted to draw complex numerical pyramid schematics in a notebook, and endorsed that God was speaking to him more over the past week. He was diagnosed with bipolar disorder 18 years ago with most recent manic episode occurring 16 years ago, resulting in a failed suicide attempt by hanging and subsequent hospitalization. Since then, the patient followed up with an outpatient psychiatrist on Risperdal $1 \mathrm{mg}$ every night (QHS) and lithium $900 \mathrm{mg}$ QHS and was otherwise noted to be highly functional and performed well at a high functioning professional. The patient had no other history
Correspondence: Hannah Muniz Castro, University of California, Irvine School of Medicine, 1001 Health Sciences Rd, Irvine, CA 92617, USA.

E-mail: hmunizca@uci.edu

Key words: self-enucleation; bipolar disorder, hyperreligiosity.

Contributions: the authors contributed equally.

Conflict of interest: the authors declare no potential conflict of interest.

Received for publication: 22 March 2017. Accepted for publication: 22 March 2017.

This work is licensed under a Creative Commons Attribution-NonCommercial 4.0 International License (CC BY-NC 4.0).

(C) Copyright H. Muniz Castro et al. 2017 Licensee PAGEPress, Italy

Mental Illness 2017; 9:7141

doi:10.4081/mi.2017.7141

of self-harming behavior.

On mental status exam he appeared well groomed with good hygiene and was dressed appropriately for the weather. Speech was within normal limits for rate, rhythm, and volume. He exhibited normal psychomotor behavior; mood was good, and affect was inappropriately flat given the severity of his injuries. He related to his examiners in a nonchalant demeanor. His thought process was linear and goal-directed as evidenced by his stating I do not feel any pain or remorse, I feel I have done the right thing. What I was supposed to do and I am cleansed now. He endorsed auditory hallucinations of God's voice, and denied visual hallucinations, paranoid ideation, and suicidal or homicidal ideations. He had no overt impairment of impulse control and was able to answer questions appropriately. His insight and judgment were impaired.

On ophthalmology exam the patient's visual acuity (VA) was no light perception (NLP) for both eyes (OU). The patient's eyes were found to have severe proptosis, extensive conjunctival lacerations and hemorrhage, and visibly avulsed extraocular muscles (Figure 1). A CT scan of the orbits demonstrated small and irregular globes, air within the orbits, and intraocular hemorrhage (Figure 2). Multiple foci of air and soft tissue stranding is also seen (Figure 3 ). The optic nerves were on stretch with posterior tenting of the globe suggesting severe optic nerve injury. Due to the optic nerve injury, the patient was started on intravenous (IV) methylprednisolone to reduce orbital edema and optic nerve inflammation 
and placed on IV and topical antibiotics. He was taken to the operating room by ophthalmology to repair the conjunctival lacerations, reattach the extraocular muscles and to limit the exposure of his eyes by detaching his lateral canthal tendons and suturing his eyelids closed with tarsorraphies (Figure 4).

Postoperatively, the patient was admitted inpatient for medical and psychiatric stabilization. The psychiatry team evaluated the patient and gave the diagnosis of bipolar mania with psychotic features, with the most recent episode constituted by hyperreligious delusions and command auditory hallucinations. When he arrived on the inpatient psychiatric unit he was disorganized, responded to internal stimuli, and expressed thoughts of self-harm such as cutting off his arm to further cleanse himself. As his lithium level was therapeutic on admission (1.22), his mood stabilizer was switched to Depakote $1500 \mathrm{mg}$ QHS and Risperdal was increased to $1 \mathrm{mg}$ twice a day (BID). An ophthalmic examination one week after surgery demonstrated NLP vision OU with nonreactive pupils $\mathrm{OU}$ and complete ophthalmoplegia. Over the course of 3 weeks he slowly gained insight to the extent of the damage he caused and expressed some regret. He was discharged in stable psychiatric condition with close outpatient psychiatric follow-up.

\section{Discussion and Conclusions}

Attempted self-enucleation is important to diagnose and manage as it can have both devastating ophthalmologic consequences. One of the most dreaded complications is complete visual loss, such as in this patient we report. Management requires assessment for visual potential, closure of wounds, and limitation of ophthalmic exposure in cases of severe proptosis. Orbital inflammation and edema can be controlled with intravenous corticosteroids if there is no concomitant head injury, as blunt head injuries were shown to have a higher mortality rate in patients receiving high dose steroids. ${ }^{7}$ Torn muscles should be reattached to circumvent permanent ophthalmoplegia. The retina should be evaluated for any tears or detachment. In cases of unilateral involvement, visual field testing should be performed as attempted self-enucleation may cause damage to the optic chiasm and affect the uninvolved eye. ${ }^{8}$ If the involved eye is damaged beyond repair, surgical enucleation may be necessary to reduce the risk of sympathetic ophthalmia, ${ }^{6}$ a rare condition of autoimmune damage against the uninvolved eye.

The medical and psychiatric literature suggest that patients who engage in attempts of self-enucleation are at risk for further self-mutilating behaviors. Dilly et al. presented a case in which a man engaged in self-enucleation of an eye which had already been enucleated via a previous attempt. ${ }^{9}$ This demonstrated that vison is not required for a psychotic patient to selfenucleate an eye. Witherspoon et al. ${ }^{10}$ demonstrated that patients who have engaged in unilateral self-enucleation are at increased risk of completing bilateral enucleation in the future. This supports the notion that recidivism is a strong component of self-enucleation behaviors even in the setting of grave consequences experienced due to a prior episode. Lack of pain and remorse have been common features of self-enucleation during psychosis and is consistent with our patient's presentation.

Cases of attempted and completed selfenucleation are rare, but require comprehensive care and interdisciplinary management between teams in emergency medicine, internal medicine, psychiatry, and ophthalmology. Our case contributes to the literature by reporting a more uncommon situation of self-enucleation attempted by a previously high-functioning patient who developed an episode of bipolar mania and acute psychosis. Furthermore, our patient became psychotic even in the setting of

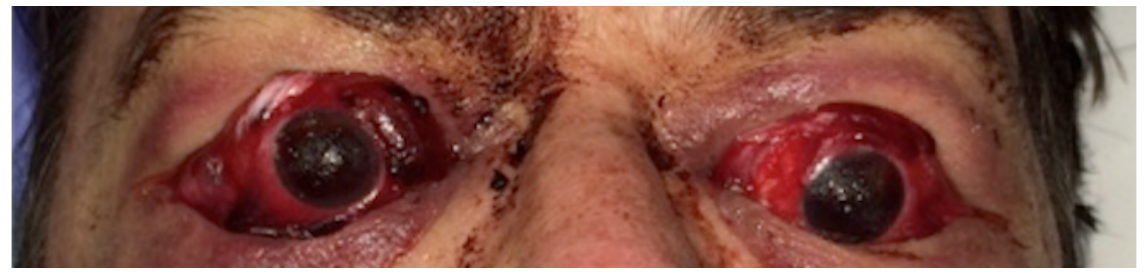

Figure 1. Appearance on presentation after attempted bilateral self-enucleation. The globes have been pulled anteriorly and display significant conjunctival lacerations and hemorrhage. Due to the anterior displacement there is severe exposure keratopathy with poor eyelid coverage of the corneas bilaterally. The superior rectus muscle is exposed and dehisced from its attachment in the right eye.

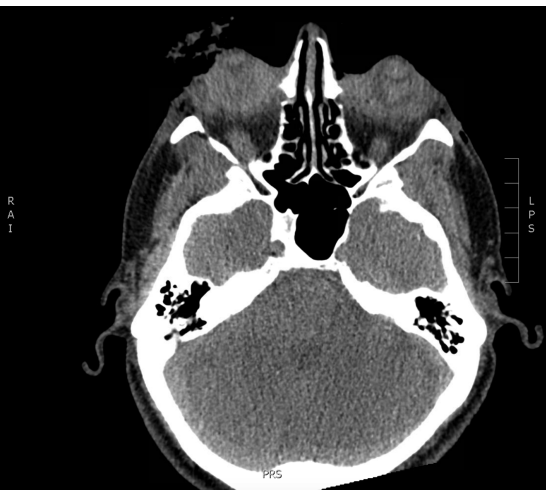

Figure 2. Axial computed tomography scan of the orbits without contrast showing small, irregular globes anteriorly displaced. There is bilateral intraocular hemorrhage and disorganization of the intraocular contents.

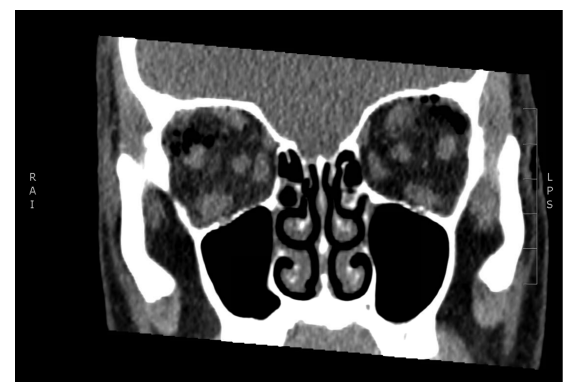

Figure 3. Coronal computed tomography scan of the orbits without contrast showing multiple foci of air and trace fat stranding within the extraconal and intraconal orbits bilaterally.

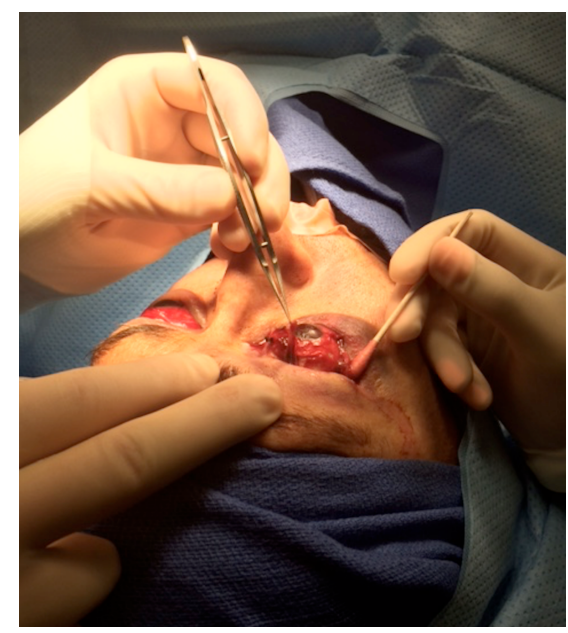

Figure 4. Appearance during intraoperative wound exploration and repair. There is severe bilateral proptosis and conjunctival lacerations. The right eye shows a dehisced superior rectus muscle from the injury. 
supratherapeutic lithium dose on admission, therefore requiring the selection of alternative mood stabilizer and increase in antipsychotic dosage. Future studies of the most effective antipsychotic and mood stabilizers and doses would greatly benefit the psychiatric literature in management of this severe self-mutilation behavior.

\section{References}

1. Zhang M, Tanaka S, Mercier M, et al. Gender and racial disparities in cases of autoenucleation. Semin Ophthalmol
2016;31:415-25.

2. Fan AH. Autoenucleation: a case report and literature review. Psychiatry (Edgmont) 2007;4:60-2.

3. Gauger EH, Sobel RK, Allen RC. Complications and outcomes after autoenucleation. Curr Opin Ophthalmol 2015;26:429-38.

4. Sophocles GD. Oedipus the King. Chicago: University of Chicago Press; 2010.

5. Holy Bible. American Standard Version. Camden: Thomas Nelson \& Sons; 2010.

6. Patton N. Self-inflicted eye injuries: a review. Eye (Lond) 2004;18:867-72.
7. Edwards P, Arango M, Balica L, et al. Final results of MRC CRASH, a randomised placebo-controlled trial of intravenous corticosteroid in adults with head injury-outcomes at 6 months. Lancet 2005;365:1957-9.

8. Wolff RS, Wright MM, Walsh AW. Attempted autoenucleation. Am J Ophthalmol 1996;121:726-8.

9 Dilly JS, Imes RK. Autoenucleation of a blind eye. J Neuroophthalmol 2001; 21:30-1.

10 Witherspoon D, Feist FW, Morris RE, Feist RM. Ocular self-mutilation. Ann Ophthalmol 1989; 21:255-9. 